\title{
Models of CD8 + Responses: 1. What is the Antigen-independent Proliferation Program
}

\author{
Rustom Antia* $\dagger$, Carl T. Bergstrom $\$ \S$, Sergei S. Pilyugin $\|$, Susan M. Kaech \\ AND RAFI AHMED
}

\begin{abstract}
$\dagger$ Department of Biology, Emory University, Atlanta, GA 30322, U.S.A. §Department of Zoology, University of Washington, Seattle, WA 98195, U.S.A. ||Department of Mathematics, University of Florida, Gainesville, FL 32611, U.S.A. and Vaccine Research Center, Emory University, Atlanta, GA 30322, U.S.A.
\end{abstract}

(Received on 11 April 2002, Accepted in revised form on 1 November 2002)

\begin{abstract}
Recent experimental results show that even brief stimulation with antigen can cause antigenspecific CD8 T-cells to undergo sustained proliferation followed by differentiation into memory cells. These results show that the dynamics of these immune responses are not governed by constant monitoring of antigen levels, but rather that following stimulation immune cells commit to a "program". At present relatively little is known about the program which governs CD8 cell proliferation and differentiation. For example, we do not know whether the program is completely specified by the initial encounter of a $\mathrm{T}$ cell with antigen, or whether it subsequently can be modified by the amount of antigen present. Nor do we know whether the entire program for $\mathrm{T}$ cell proliferation and differentiation resides within the $\mathrm{T}$ cell itself, or whether some component(s) of the program are determined by cells or molecules external to the CD8 cell. In this paper we construct simple mathematical models which incorporate antigen-independent proliferation and differentiation of CD8 cells during acute infections. We use these models to determine what characteristics the program must have in order to be consistent with the existing data on the dynamics of CD8 responses, and in particular to answer the questions posed above. Our results suggest that the program is not completely defined by the initial encounter of $\mathrm{T}$ cell with antigen but may be augmented by exposure to antigen in a brief window shortly after infection; furthermore, parts of the program may reside external to the T-cells. Finally we examine some of the consequences of the "program" for pathogen-host coevolution.
\end{abstract}

(C) 2003 Published by Elsevier Science Ltd.

\section{Introduction}

How are specific immune responses generated and regulated? The theory of clonal selection (Burnet, 1959) postulates that antigen-specific immune responses are generated by expansion of a small number of antigen-specific cells to a

$\$$ Contributed comparably to this paper.

*Corresponding author. Tel.: +1-404-727-1015; fax: $+1-404-727-2880$.

E-mail address: rantia@emory.edu (R. Antia). population sufficient to control the pathogen. We begin by briefly reviewing the basic features of the dynamics of immune responses during acute and persistent infections, focusing on the acute CD8 T cell responses, ${ }^{*}$ following infection

\footnotetext{
* We consider the CD8 response because it has been extensively studied in a quantitative manner and it can be generated independently of other (T-helper and antibody) specific responses, thus making it potentially easier to describe in terms of mathematical models.
} 
with viruses such as the lymphocytic choriomeningitis virus (LCMV) during which the virus is rapidly cleared by the immune response within the first week following infection.

The dynamics of the CD8 response during an acute infection has three major phases: an expansion phase, a contraction phase and a memory phase (see Fig. 1) (Murali-Krishna et al., 1998; Ahmed \& Gray, 1996). Following infection of a cell, viral epitopes (short peptides derived from the virus and bound to MHC class I molecules) are expressed on the surface of infected cells. CD8 cells specific for these epitopes expand from very low numbers $(\approx 50$ 100 cells per epitope in the spleen of a mouse) to populations of up to $10^{7}$ cells. Responses to the different epitopes may differ in the time at which they are initiated and in their rate of growth. The CD8 cell expansion stops some days after the virus has been controlled and subsequently the CD8 responses to the various epitopes decline synchronously by apoptosis and about $5-10 \%$ of the population differentiates into memory cells.

Current models of the dynamics of CD8 and other immune responses are formally analogous to predator-prey models in ecology. In these models, the rate of proliferation of immune cells (predators) is continually updated according to the density of the pathogen (prey) present in the body (ecosystem) (Bell, 1970; Nowak \& Bangham, 1996; DeBoer \& Perelson, 1995; Oprea \& Perelson, 1996; Pilyugin et al., 1997; Wodarz et al., 1998; DeBoer et al., 2001; Bocharov, 1998). We hereafter refer to these as predatorprey type models. The differentiation of CD8 cells is modeled by introducing subpopulations of naive, activated/effector and memory cells (Bocharov, 1998; Wodarz et al., 1998; DeBoer et al., 2001). These models reproduce many dynamic features of the CD8 response, including the basic expansion-contraction dynamics, generation of immunodominance (responses against different epitopes have different magnitudes), and the synchrony in the contraction phase of the response. However, predator-prey models fail to explain several key features of the specific immune response. First, experimental studies show that the immune response continues to rise even after pathogen clearance. For example, the peak in the CD8 response to the lymphocytic choriomeningitis virus (LCMV) can occur several days after the clearance of the pathogen (Murali-Krishna et al., 1998). Second, the immunodominant and subdominant responses appear to behave independently of clearance of the pathogen (see below) and independent of each other-removal of the dominant epitope

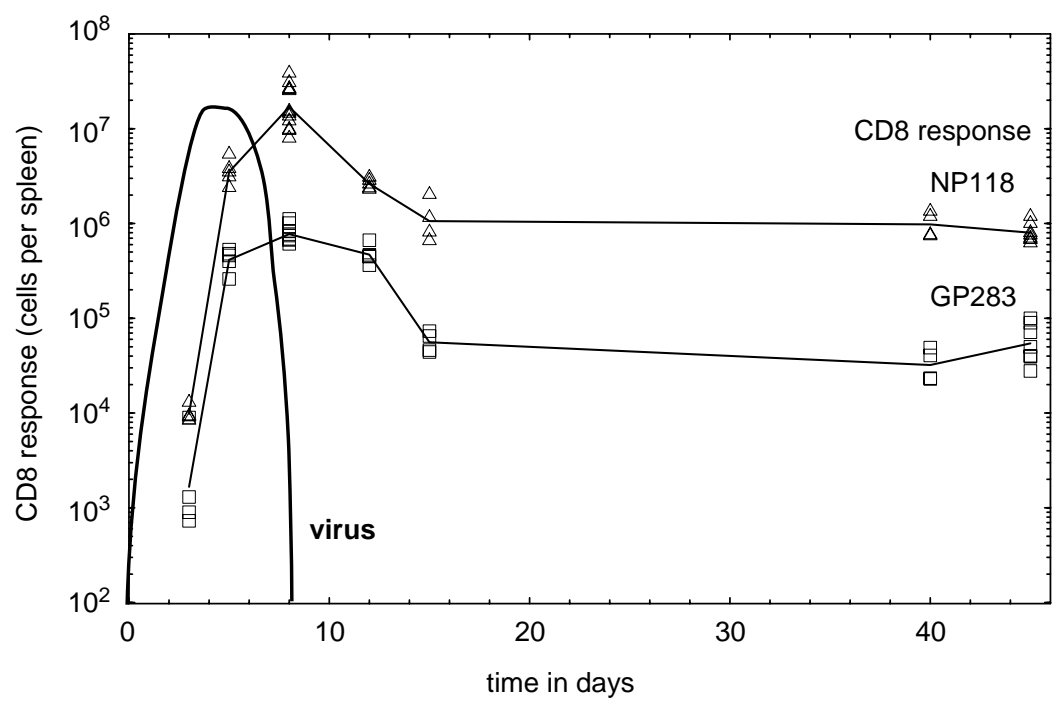

FIG. 1. Dynamics of the CD8 response. Dynamics of CD8 + cells and virus during acute infections of BALB/c (H-2 $\left.{ }^{d}\right)$ mice with LCMV Armstrong strain (Murali-Krishna et al., 1998). The immunodominant (NP 118) and subdominant (GP 283) epitopes are represented by triangles and diamonds, respectively. Virus is indicated schematically. 
does not always appear to result in a compensatory increase in the number of cells of the other (subdominant) responses (Vijh et al., 1999; van der Most et al., 1997).

A set of recent experimental studies (Mercado et al., 2000; Wong \& G. Pamer, 2001; van Stipdonk et al., 2001; Kaech \& Ahmed, 2001; Bevan \& Fink, 2001) offer even more direct evidence that the predator-prey framework for immune response modeling must be modified. These studies show that following brief antigenic stimulation, naive T-cells are committed to continue proliferating and subsequently to differentiate into memory cells in the absence of further stimulation. Mercado et al. (2000) monitored the dynamics of CD8 responses following infection of mice with Listeria monocytogenes. They found that treatment of the mice with an antibiotic 1 day after infection resulted in rapid elimination of the bacteria but relatively little change in the CD8 immune response, suggesting that the immune response was "programmed" during the first day following infection. The antigen-independent nature of the proliferation was supported by a subsequent study by this group (Wong \& G. Pamer, 2001) which showed that brief $(2.5 \mathrm{hr})$ stimulation with antigen was sufficient to cause antigen-specific $\mathrm{CD} 8+$ cells to undergo up to 8 rounds of proliferation in vitro. Stipdonk et al. (2001) used engineered antigen-presenting cells that expressed both antigen and costimulatory molecules to stimulate transgenic $\mathrm{T}$ cells specific for the antigen. They found that as little as $2 \mathrm{hr}$ of exposure were sufficient to stimulate the specific naive CD8 cells and that subsequently - even in the absence of antigen - these CD8 cells underwent many rounds of division, and acquired cytotoxic effector capabilities. Kaech \& Ahmed (2001) examined the dynamics of transgenic antigen-specific cells following stimulation by varying doses of antigen. They found that the antigen dose predominantly affected the number of antigen-specific cells recruited into the response. Following recruitment, the cells continued to divide and differentiate into memory cells that were capable of conferring protective immunity.

These new experimental studies convincingly demonstrate the existence of an antigenindependent proliferation program. In doing so, they force us to change the earlier "predator-prey" framework commonly used to model immune responses.

However, these studies give us only a relatively limited idea of what rules govern the dynamics of immune cells. They do not, for example, tell us whether this "program" is completely specified by the initial encounter of a $\mathrm{T}$ cell with antigen, or whether it can be subsequently modified by the amount of antigen present. Nor do we know whether the entire program for $\mathrm{T}$ cell proliferation and differentiation resides in the $\mathrm{T}$ cell itself or whether some component(s) of the program are determined by cells or molecules external to the CD8 cell. The objectives of this report are as follows: (i) to construct mathematical models which describe the dynamics of immune responses which have an antigen-independent expansion phase, (ii) to use this framework together with data (from the recent papers mentioned above as well as from earlier quantitative studies of the immune response during acute infections) to discriminate between different possible programs for the generation of immune responses; (iii) to identify key experimental measurements that will help us understand the generation of immune responses and (iv) to consider the consequences of the program for pathogen-host coevolution and self-non-self discrimination.

As the current data on program pertains to the primary CD8 response, in this paper our models focus exclusively on the primary CD8 response during acute infections. In these circumstances the pathogen is cleared prior to the generation of memory, and we do not consider the immune program during either secondary responses or during persistent infections. At the present time we are interested predominantly in discriminating between alternative models, and do so by determining which models generate dynamics which is qualitatively consistent with experimental observations.

\section{What is the Immune Program?}

\subsection{THE SIMPLEST PROGRAM}

We first consider the simplest possible model of a CD8 cell proliferation program: following initial stimulation, the CD8 cell progresses 
through a fixed program of expansion, contraction, and differentiation into memory cells. We call such a program a "strict" program: it is not altered in any way by subsequent exposure to antigen. We first consider how such a program might be modeled, and then consider the consequences of this strict program for the dynamics of acute infections.

\subsubsection{Modeling a Strict Program}

There are a number of ways in which the program could be described quantitatively, and in Appendix A we describe two possible models in detail. The basic results described in this paper hold regardless of which of these two models we use to describe the program. The simulations shown in the figures employ Model A, described in Appendix A. For simplicity we focus on the program for the dynamics of the expansion and contraction phase.

In both models we begin with all cells in the resting or naive state $N$. Upon stimulation, which occurs at a rate dependent on the rate of encounter with antigen, these cells are recruited into the response. In Model A, once recruited the subsequent behaviors of cells depend on the time elapsed $(\tau)$ following stimulation. In this model, recruited cells undergo antigen-independent expansion for a fixed amount of time followed by contraction and differentiation into memory cells. In Model $\mathrm{B}$, the rate of proliferation depends on the number of divisions the cells have undergone. During the initial divisions the proliferation rate exceeds their death rate and in subsequent divisions the death rate exceeds the proliferation rate.

The models are described in Fig. 2. Details of these model as well as their extension to incorporate multiple epitopes is described in Appendix A. As the basic results obtained with both models are similar in the subsequent sections we use one of these models (Model A) for the simulations.

\subsubsection{Results of a "Strict" Program}

The dynamics of a strict program are illustrated in Fig. 3. At first glance, this simple

\section{Conventional Model}

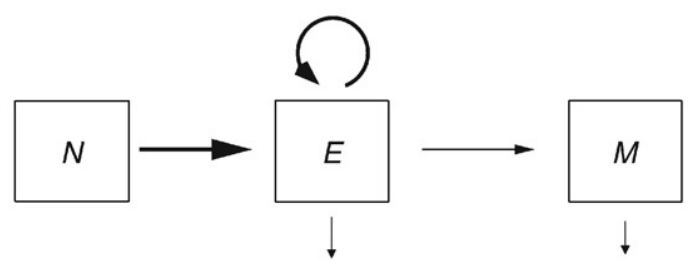

Program A (PDE model)

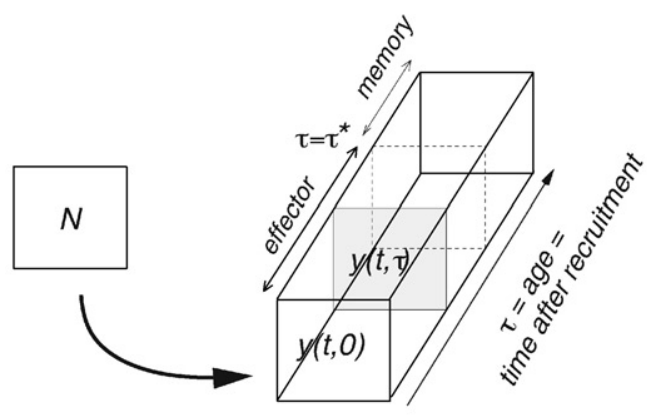

Program B (ODE model)

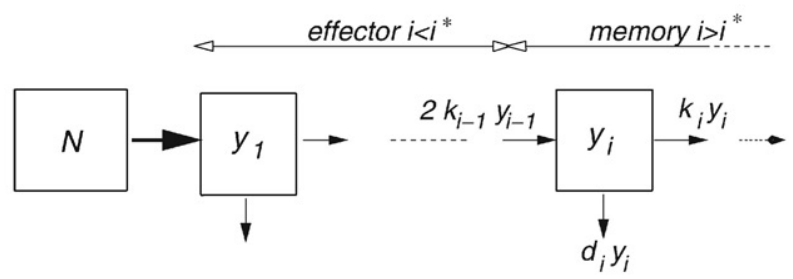

FIG. 2. Outline of models. We outline our models for CD8 responses during acute infections. Antigen-dependent processes are shown by the thick arrows and antigenindependent processes are shown by the thin arrows. The conventional model which lacks any antigen-independent proliferation as well as the program models which incorporate antigen-dependent proliferation are shown above. In the conventional model both recruitment from the naive compartment $N$ and proliferation are antigen dependent, while in the "strict program" only the recruitment is antigen-dependent. We show two ways in which the antigen-independent proliferation can be modeled. In the PDE model antigen-independent proliferation, death and differentiation from effector to memory cells is a function of time $\tau$ after recruitment. In the ODE model antigen-independent proliferation, death and differentiation from effector to memory cells is a function of the number of divisions $i$ the cells have undergone.

model reproduces the basic features of the CD8 immune response: namely, the expansion, contraction and memory phases [Fig. 3(A)]. With respect to regulation of the response and the generation of immunodominance 

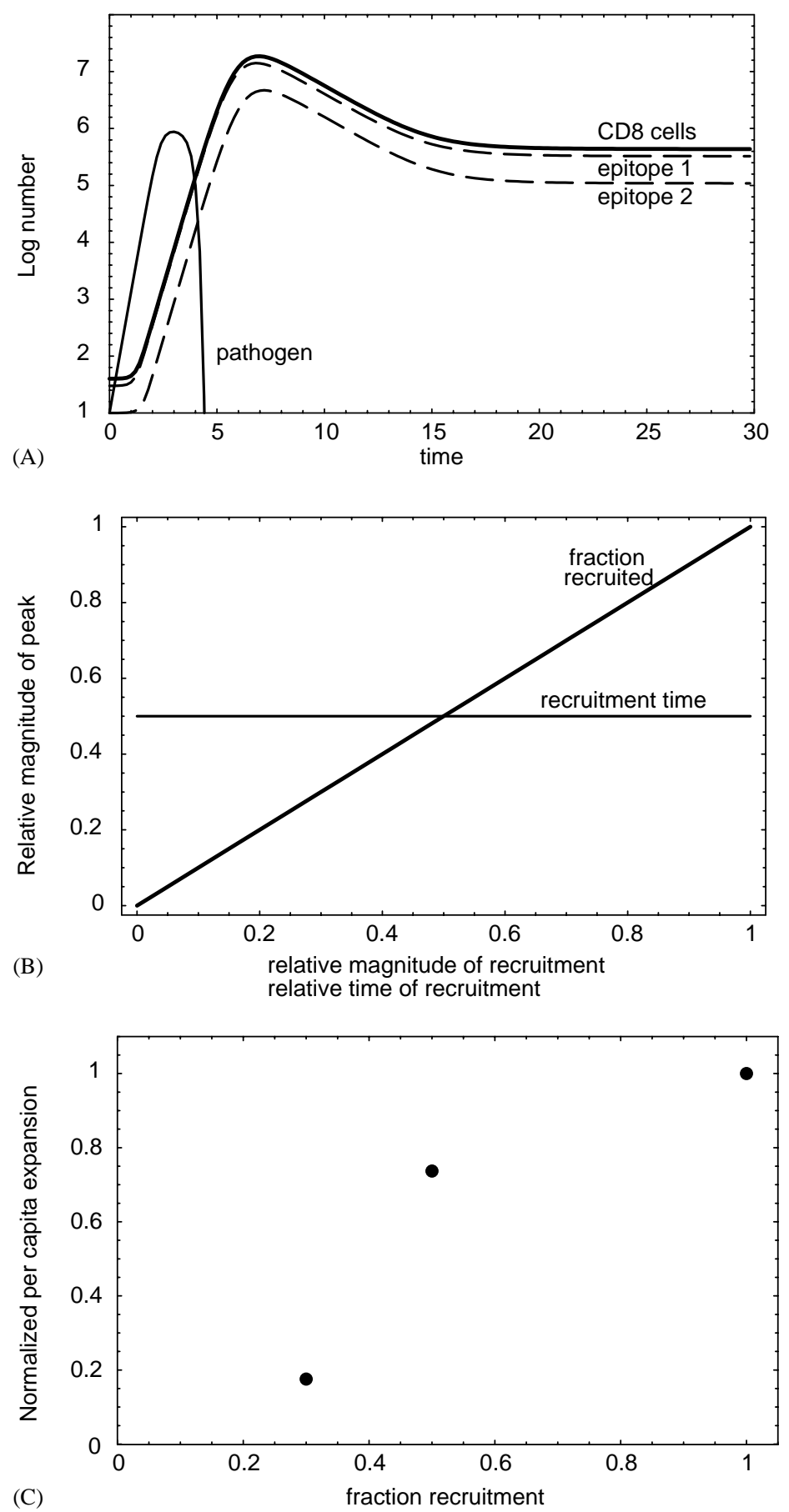

FIG. 3. Strict program model. (A) Predicted dynamics of the CD8 response to two epitopes. The pathogen and total CD8 response are shown in solid lines and the CD8 responses to individual epitopes are shown in the dotted lines as indicated. Note that differences in the time of recruitment give rise to differences in the time of the peak of the response. (B) We examine how immunodominance may be generated by plotting the relative magnitude of the response to an epitope as a function of the magnitude of recruitment (i.e. the number of cells recruited) and timing of this recruitment. We find that the simple model predicts that the magnitude of the response to an epitope is directly proportional to the number of cells recruited and does not depend on the timing of this recruitment. (C) Re-plotting the data described in Kaech \& Ahmed (2001) suggest that the per capita expansion (relative magnitude of the response (normalized with respect to the maximum magnitude when all cells are recruited) divided by the fraction of cells recruited) increases with increasing fraction recruitment. This suggests that infections with higher doses of the pathogen (which also take longer to be cleared) result in greater recruitment and also greater proliferation on a per cell basis. See Appendix A for details and parameters. 
[Fig. 3(A) and (B)], we find that the strict program model makes the following predictions:

1. If the window of recruitment is short the magnitude of the response is determined by number of cells recruited. Because severe infections by exponentially replicating pathogens (such as LCMV) result in nearly simultaneous recruitment of all cells specific to a given epitope, the magnitude of the response will simply be proportional to the frequency of precursor cells specific to that epitope.

2. Differences in timing of recruitment of cells specific to different epitopes will result in different timing of the peak of the responses to each epitope-but the peak magnitude of the response will not be affected. (By item (1) above the peak magnitude is directly proportional to the precursor frequency.) This is shown in Fig. 3(B).

3. Responses to the different epitopes are independent. Thus removal (or any change) of the response to one epitope will not affect the magnitude or time of responses to the other epitopes. Simulations (not shown) confirm this result.

We now attempt to bring each of these predictions into contact with experimental observations.

1. A strict program predicts that the magnitude of the response is directly proportional to the number of cells recruited into the response, i.e. the per capita expansion of recruited cells is constant. If, on the other hand, the program is not strict and the expansion of recruited cells can be augmented by the magnitude and duration of antigenic stimulation then we expect more prolonged and larger stimulation will result not only in a greater fraction of cells recruited but also a larger per capita expansion of the recruited cells. Kaech \& Ahmed (2001) have shown that when the magnitude of the infection increases the fraction of CD8 cells recruited increases as does the total response. A plot of the per capita expansion vs. recruitment [Fig. 3(C)] suggests that the per capita expansion infections with higher doses of the pathogen (which also take longer to be cleared) result in greater recruitment and also greater proliferation on a per cell basis.

2. A strict program predicts that immunodominance depends principally on the number of epitope-specific cells recruited. Other factorssuch as differences in the timing of initiation of the responses to the different epitopes - should play a minor role in the generation of immunodominance. The experimental data suggest otherwise: additional factors, such as the time of recruitment, also contribute to immunodominance (Yewdell \& Bennink, 1999; DeBoer et al., 2001). Furthermore, the data suggests that the peak magnitudes of the responses to different epitopes are synchronous (Murali-Krishna et al., 1998; DeBoer et al., 2001), which is also inconsistent with a strict program.

3. A strict program is consistent with the experiments showing the lack in compensation of the subdominant responses when the immunodominant epitope is removed (Vijh et al., 1999; van der Most et al., 1997). (See Section 3.1 for a more critical discussion.)

We conclude that the strict program is inconsistent with a number of experimental results. The simple program needs to be modified. We consider possible ways of modifying the program in the next section.

\subsection{INCORPORATING ANTIGEN-DEPENDENT EXPANSION}

In this section we examine how the magnitude of the response can be influenced by ongoing antigenic stimulation during the course of the response. We begin by considering a window of antigen-dependent expansion prior to the antigen-independent program. During this window if antigen is present proliferation is antigendependent, and subsequent to the end of the window, (or clearance of the antigen if that comes first) there is antigen-independent proliferation as described in the previous section. Using simple modifications of the "strict program" to incorporate a window of antigendependent expansion (results not shown) suggested that for large infections (such as LCMV and Listeria monocytogenes) the window must end before the clearance of the pathogen. The explanation is very simple, this is required so 
that: (i) the duration/magnitude of exposure to the pathogen is able regulate the magnitude of the expansion, but (ii) there must be a lack of compensation in the magnitude of the response to the subdominant epitope when the response to the dominant epitope is experimentally removed.

We consider two alternative models, in which the duration of this window is determined by an internal signal, or an external signal, respectively. In the internal signal model, each $\mathrm{T}$ cell proliferates in an antigen-dependent manner for a fixed duration after its recruitment. In the external signal model, the window of antigendependent proliferation is ended by a common external signal, such as a cytokine in the environment. By extending our quantitative model of the strict program to incorporate these two types of antigen-dependent proliferation, we can explore the characteristics of these models and in particular their differences. We show how this can help in discriminating between these models.

As one might expect [see Fig. 4(A) and (B)], both models generate the basic expansion, contraction and memory phases. For the reasons described at the beginning of this section (i.e. provided the period of antigen-dependent expansion is short compared to the time of clearance of the pathogen) both models predict
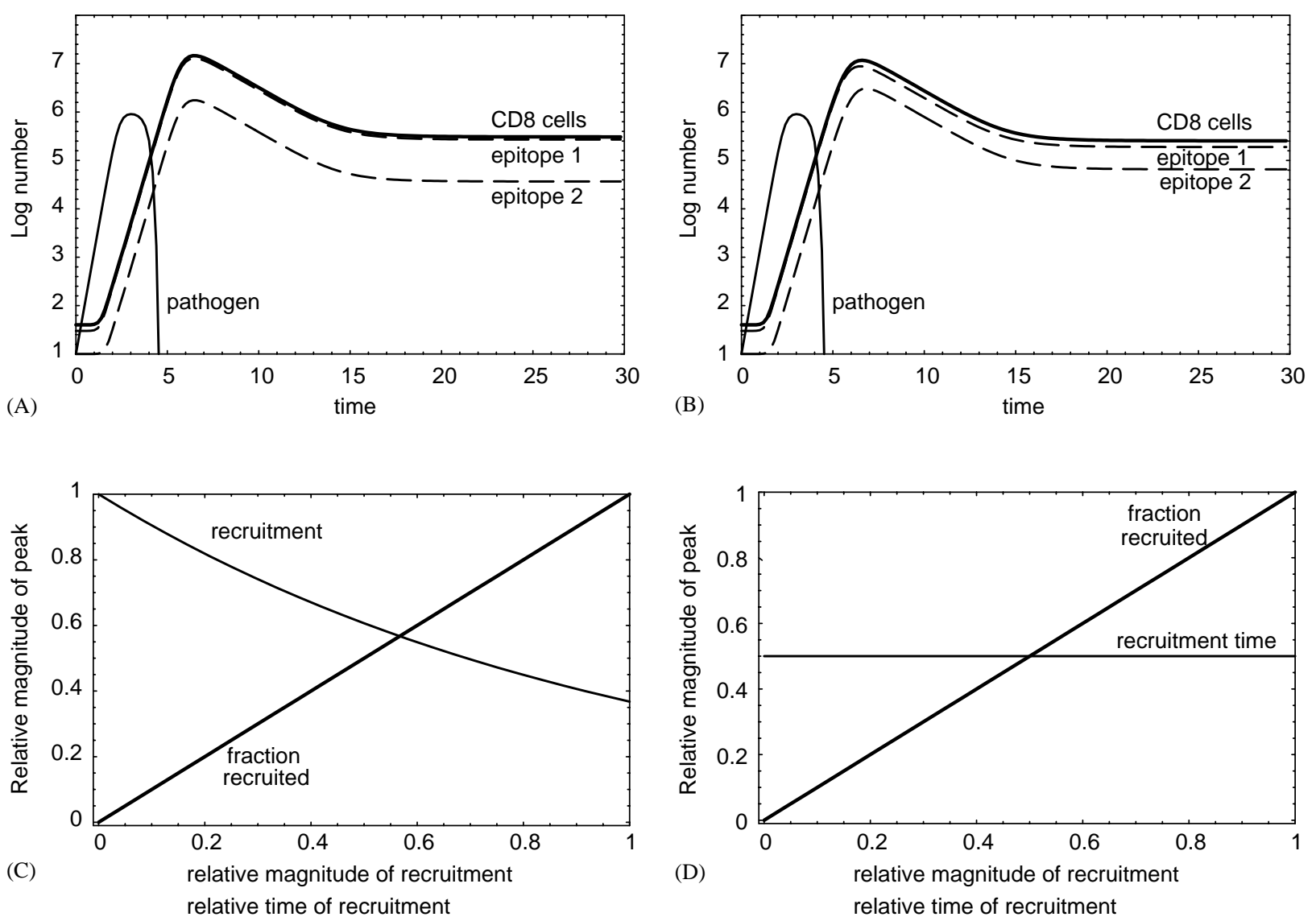

FIG. 4. Incorporating antigen-dependent proliferation to the program. We incorporate a period of antigen-dependent proliferation prior to the antigen-independent expansion program. In (A) and (B) we show representative simulations of the dynamics of infection when the timing of antigen-dependent expansion is external to and internal to the T cells, respectively. In (C) and (D) we examine how immunodominance may be generated by plotting the relative magnitude of the response to an epitope as a function of the magnitude of recruitment (i.e. the number of cells recruited) and timing of this recruitment. For the external signal model (C) we find that the magnitude of responses to an epitope depends linearly on the magnitude of recruitment and varies inversely with the average time of recruitment. For the internal signal model (D) we find that the magnitude of the response to an epitope depends only on the magnitude of recruitment and not the time of recruitment of the cells. See Appendix A for details and parameters. 
that the responses to the different epitopes are independent (removal of one epitope does not substantially alter the response to other epitopes). More careful examination of the dynamics of immunodominance reveals the following differences between the two models [see Fig. 4(C) and (D)]:

1. Differences in the precursor frequency and differences in the time of initiation of the response to different epitopes contribute to immunodominance in the external signal model. Only differences in the precursor frequency contribute to immunodominance in the internal signal model [Fig. 4(C) and (D)].

2. Differences in the time of initiation of the response to different epitopes gives rise to similar differences in the time of the peak of these responses in the internal signal model. The peak responses to different epitopes are synchronous in the external signal model.

As in the previous section (Section 2.1) we examine if the data on the dynamics of CD8 the responses to different epitopes and the generation of immunodominance are consistent with internal and external programs.

1. Data on immunodominance strongly suggest that not only precursor frequency, but also differences in the timing of initiation of the response to different epitopes, may contribute to immunodominance (Yewdell \& Bennink, 1999; DeBoer et al., 2001).

2. The data on the dynamics of the CD8 responses are consistent with synchrony in the peak of the responses to different epitopes. However, it is not presently possible to determine if there are small departures from synchrony (Murali-Krishna et al., 1998; DeBoer et al., 2001).

To summarize our while our results suggest (provisionally) that the antigen-dependent component of the program might be regulated by an external signal.

\section{Discussion}

We have constructed simple mathematical models to describe antigen-independent proliferation and differentiation of CD8 cells during acute infections. By bringing these models into contact with experimental observations of CD8 responses, we are able to discriminate between alternative programs for the generation of immune responses. Our results, summarized in Table 1, suggest that a strict program should be rejected. We have suggested one alternative model, namely that the expansion program may be regulated by signals external to the $T$ cell. Clearly we have not and indeed cannot consider all possible models. An alternative model which is consistent with the experimental data we consider has the APC deliver a program to the $\mathrm{T}$ cell on encounter with the duration of the program decreasing with time after infection.

In this section we (i) suggest how the models can be brought into closer contact with experiments, (ii) consider some of the possible selective advantages of having a prolonged antigenindependent expansion phase, and (iii) consider ways in which the models can be refined/ extended.

\subsection{EXPERIMENTAL TESTS}

In this section we examine several ways in which the models can be experimentally tested. The first involves determining the interaction between the antigen-dependent and antigenindependent components to the response, the second involves more careful consideration of the assumptions on which the model is based; the third involves experimental tests of the predictions of the model.

Our models suggest that we need to better describe the antigen-dependent and antigenindependent components to the response, or the autonomous and antigen-dependent components of the immune program. This problem is analogous to that in developmental biology where part of the "developmental program" may reside within the cell and part may reside in the environment of the cell (Wolpert, 1991). As in developmental biology this problem may be addressed by transfer of labelled cells obtained from mice at a given stages of the immune response into mice at a different stage of the response. We note that the CFSE label to mark the transferred cells should allow determination of the subsequent proliferation history of these cells. 
TABLE 1

We summarize the ability of the different models to describe the general features of immune responses as well as of immunodominance

\begin{tabular}{|c|c|c|c|c|}
\hline & Predator-prey & Strict program & Internal signal & External signal \\
\hline \multicolumn{5}{|l|}{ General features } \\
\hline $\begin{array}{l}\text { 1. Expansion, contraction and } \\
\text { memory phases }\end{array}$ & Yes & Yes & Yes & Yes \\
\hline $\begin{array}{l}\text { 2. Antigen-independent and } \\
\text { expansion phase }\end{array}$ & No & Yes & Yes & Yes \\
\hline $\begin{array}{l}\text { 3. Magnitude of expansion } \\
\text { regulated by antigen }\end{array}$ & Yes & No & Yes & Yes \\
\hline \multicolumn{5}{|l|}{ Epitope-specific responses } \\
\hline $\begin{array}{l}\text { 4. Independence of responses } \\
\text { to different epitopes }\end{array}$ & No & Yes & Yes & Yes \\
\hline 5. Synchrony at peak & Yes & No & No & Yes \\
\hline \multicolumn{5}{|l|}{$\begin{array}{l}\text { 6. Immunodominance } \\
\text { determined by }\end{array}$} \\
\hline (a)precursor frequency & Yes & Yes & Yes & Yes \\
\hline (b)timing of recruitment & Yes & No & No & Yes \\
\hline
\end{tabular}

The assumptions on which the model is based which need to be more accurately determined include the following. (i) To what extent there is synchrony in the peak of the responses to different epitopes. (ii) The extent to which differences in timing of recruitment and the extent of recruitment affect the magnitude of the response and immunodominance. (iii) The extent to which there is compensation in the subdominant response following removal of the dominant response. There is some uncertainty to the extent of compensation: some studies suggest almost no compensation (van der Most et al., 1997, Vijh et al., 1999), while others are consistent with almost complete compensation (Weidt et al., 1998). Specifically two issues should be addressed. First the papers showing lack of compensation (van der Most et al., 1997; Vijh et al., 1999) make their measurements close to the peak of the primary response while the compensation may, at least in conventional predator-prey type models, be expected to delay the timing of the peak. Second, the measurement of CTL should be made using MHC-tetramers or intracellular cytokine assays such as those used by Vijh et al. (1999) which are more accurate than the earlier ex vivo CTL-killing assays used by van der Most et al. (1997) and Weidt et al. (1998) that are not able to distinguish between changes in killing activity per cell and changes in the numbers of epitopespecific CD8 cells.

The predictions of the model include the following. (i) The time to the peak of the response can vary from, at minimum, the duration of the antigen-dependent expansion program to at maximum the duration of the window of antigen-dependent stimulation plus the duration of antigen-independent expansion program. (ii) Following infections of very short duration which are cleared prior to the end of the window of antigen-dependent proliferation we predict that there should be substantial compensation in the magnitude of the subdominant response following removal of the dominant response, and the response should peak slightly earlier. In the context of the last prediction we note that most of the current data on the dynamics of the response following infection is obtained following infections with pathogens such as LCMV and Listeria which induce massive proliferative responses, and much less is known about pathogens which elicit smaller responses.

\subsection{WHY HAVE A PROGRAM?}

From a functional perspective, what are the advantages and disadvantages of employing an immune response which commits to a fixed 
program rather than constantly updating its response by tracking the pathogen? In this section, we address this question from an evolutionary (and predominantly adaptationist) viewpoint. At first glance, continual updating might seem to be a more efficient way to deal with pathogen challenge, in that the immune response can be more finely tuned and optimized to the current infection. However, viruses and bacteria employ an extensive array of mechanisms that serve to subvert immune responses, and potentially this sensing apparatus (Gooding, 1992; Evans \& Desrosiers, 2001). This sort of subversion could be avoided if an antigen-independent program is set before the pathogen has the opportunity to alter it, i.e. early in infection while pathogen density is low. Program responses, while less efficient, are likely to be more robust in that they will be less prone to interference from the pathogen.

Given a programmed response, what would we expect its features to be? Since the pathogen environment is highly variable (unpredictable) and there is no fine-tuning possible, the expansion program must err on the side of caution, typically overshooting the necessary number of CD8 cells required to clear the pathogen. This is indeed what is observed. CD8 proliferation continues well beyond the point of pathogen clearance in many infections. Immunodominance experiments illustrate just how markedly the immune system overshoots most infections: following removal of the immunodominant response to Listeria, the subdominant responses do not appear to increase substantially but nevertheless are able to control the infection (Vijh et al., 1999).

\subsection{FURTHER STUDIES}

This model is a very first step in describing the programmed proliferation and differentiation of immune cells. It can be extended in a number of ways, some of which we briefly list here.

1. Generate a cell-cycle based model for the program. Cell proliferation is best considered in terms of the cell cycle and proliferation can be described quantitatively in terms of progression through the G1, S, G2, and M stages of the cell cycle. Smith \& Martin (1973) have shown that to a first approximation movement from $\mathrm{G} 1$ to $\mathrm{S}$ is a stochastic event, and subsequent progression through $\mathrm{S}, \mathrm{G} 2$ and $\mathrm{M}$ is determinate (takes a fixed period of time). Thus a cell-cycle model would involve incorporating elements of both the models described in Appendix A. It involves stochastic recruitment into division (Model B) and determinate progression through $\mathrm{S}, \mathrm{G} 2$ and M (Model B).

2. Explicitly consider the program in terms for processes of cell division and cell death which underly the changes in the total population which we have described in this paper.

3. Incorporate a lag of about one day (VeigaFernandes et al., 2000; van Stipdonk et al., 2001) following recruitment of naive cells during which the cells do not proliferate.

4. In the current model effector function and memory function change with the age of the cell in a stepwise manner (at age $\tau=\tau^{*}$ in Model A and at division $n=a+b$ in Model $\mathrm{B}$, see Appendix). We need to consider a more gradual change with differentiation of the cell and determine whether differentiation into memory is determinate at a fixed time or stochastic.

5. Explicitly consider the program for memory cells. This would incorporating the recruitment of memory cells and determining how naive and memory cells, and possibly how memory cells of different "ages" differ in their rate of recruitment into the response and how the antigendependent and antigen-independent components of the program differ for naive and memory cells.

6. Consider how the presence of antigen during the contraction phase may result in the generation of energy rather than memory cells, and apply this to consider persistent infections in more detail. This will be critical in modeling persistent infections.

7. Consider the how antigen-independent proliferation alter the dynamics of other immune cell populations such as $\mathrm{CD} 4+\mathrm{T}$ cells and B cells.

We thank M. Tanaka, D. Genereux and V. Ganusov for their comments. This work was supported by NIH grants R29 GM 54268 and R01 AI 493334 to R. Antia, and AI 30048 to R. Ahmed. 


\section{REFERENCES}

Ahmed, R. \& Gray, D. (1996). Immunological memory and protective immunity: understanding their relation. Science 272, 54-60.

Antia, R., Koella, J. \& Perrot, V. (1996). Models of the within-host dynamics of persistent mycobacterial infections. Proc. R. Soc. (London) Series B 263, 257-263.

BeLl, G. (1970). Mathematical model of clonal selection and antibody production. Nature 228, 739-744.

Bevan, M. J. \& Fink, P. J. (2001). CD8+ response on autopilot. Nat. Immunol. 2, 381-382.

Bocharov, G. A. (1998). Modelling the dynamics of LCMV infection in mice: conventional and exhaustive CTL responses. J. theor. Biol. 192(3), 283-308.

Burnet, F. M. (1959). The Clonal Selection Theory of Acquired Immunity. Cambridge: Cambridge University press.

DeBoer, R. J., Oprea, M., Antia, R., Murali-Krishna, K., Ahmed, R. \& Perelson, A. S. (2001). Recruitment times, proliferation and apoptosis rates during the CD8 $+\mathrm{T}$ cell response to LCMV. J. Virol. 75, 10663-10669.

DeBoer, R. J. \& Perelson, A. S. (1995). Towards a general function describing $\mathrm{T}$ cell proliferation. J. theor. Biol. 175, 567-576.

Evans, D. T. \& Desrosiers, R. C. (2001). Immune evasion strategies of the primate lentiviruses. Immunol. Rev. 183, $141-158$.

Gooding, L. R. (1992). Virus proteins that counteract host immune defences. Cell 71, 5-7.

Kaech, S. \& Ahmed, R. (2001). Memory CD8 + T cell differentiation: initial antigen encounter triggers a developmental program in naive cells. Nat. Immunol. 2 , 415-422.

Mercado, R., Vijh, S., Allen, S. E., Kerksiek, K., Pilip, I. M. \& PAmer, E. G. (2000). Early programming of $\mathrm{T}$ cell populations responding to bacterial infection. J. Immunol. 165(12), 6833-6839.

Murali-Krishna, K., Altman, J. D., Suresh, M., Sourdive, D. J. D., Zajac, D. J. D., Miller, J. D., Slansky, J., \& Ahmed, R. (1998). Counting antigenspecific CD8 $+\mathrm{T}$ cells: a re-evaluation of bystander activation during viral infection. Immunity $\mathbf{8}$, 177-187.

Nowak, M. A. \& Bangham, C. R. M. (1996). Population dynamics of immune responses to persistent viruses. Science 272, 74-79.

Oprea, M. \& Perelson, A. S. (1996). Exploring the mechanisms of primary antibody responses to $\mathrm{T}$ celldependent antigens. J. theor. Biol. 181, 215-236.

Pilyugin, S. S., Mittler, J. \& Antia, R. (1997). Modelling T-cell proliferation: an investigation of the consequences of the Hayflick limit. J. theor. Biol. 186, $117-129$.

Smith, J. A. \& Martin, L. (1973). Do cells cycle? Proc. Natl. Acad. Sci. U.S.A. 70(4), 1263-1267.

van der Most, R. G., Sette, A., Oseroff, C., AlexAnder, J., Southwood, S., Sidney, J., Chesnut, R. W., Ahmed, R. \& Sette, A. (1997). Uncovering subdominant cytotoxic T-lymphocyte responses in Lymphocytic choriomeningitis virus-infected BALB/c mice. J. Virol. 71, 5110-5114. van Stipdonk, M. J. B., Lemmens, E. E. \& SchoenberGER, S. (2001). Naive CTLs require a single brief period of antigenic stimulation for clonal expansion and differentiation. Nat. Immunol. 2, 415-422.

Veiga-Fernandes, H., Walter, U., Bourgeois, C., Mclean, C. \& Rocha, B. (2000). Response of naive and memory CD8 $\mathrm{T}$ cells to antigen stimulation in vivo. Nat. Immunol. 1, 47-53.

Vijh, S., Pilip, I. M. \& Pamer, E. G. (1999). Noncompetitive expansion of cytotoxic $\mathrm{T}$ lymphocytes specific for different antigens during bacterial infection. Infect. and Immun. 67, 1303-1309.

Weidt, G., Utermohlen, O., Heukeshoven, J., LehmannGrube, F. \& Deppert, W. (1998). Relationship among immunodominance of single CD8 T Cel epitopes, virus load, and kinetics of primary antiviral CTL response. J. Immunol. 160, 2923-2931.

Wodarz, D., Klenerman, P., Nowak, M. A. (1998). Dynamics of cytotoxic T-lymphocyte exhaustion. Proc. R. Soc. (London) Series B 265, 191-203.

Wodarz, D., May, R. M., NowaK, M. A. (2000). The role of antigen-independent persistence of memory cytotoxic T lymphocytes. Int. Immunol. 12(4), 467-477.

Wolpert, L. (1991). The Triumph of the Embryo. Oxford: Oxford University Press.

Wong, P. \& Pamer, G. E. (2001). Antigen-independent CD8 T cell proliferation. J. Immunol. 166(10), 5864-5868.

Yewdell, J. W. \& BenNinK, J. R. (1999). Immunodominance in major histocompatibility complex class Irestricted lymphocyte responses. Annu. Rev. Immunol. 17, 51-88.

\section{Appendix A}

In this appendix we consider two alternative formulations for describing a strict program, Model A and Model B. At present both are broadly consistent with the experimental observations. In the simulations described in the paper we have considered model A. As mentioned in the Section 1, in this paper we limit our discussion to the dynamics of the primary CD8 response during acute infections. During these infections the pathogen is cleared rapidly, prior to the generation of immune memory. We therefore do not, at this stage consider the restimulation of memory cells.

In both models $\mathrm{A}$ and $\mathrm{B}$, we track the following state variables which change over time $(t)$ : the number of naive precursor cells $N$, the pathogen density $P$, the number of CD8 effector cells $E$, and the number of CD8 memory cells $M$. The total number of responding CD8 cells are thus given by $Y=E+M$. In both cases, we begin with all cells in the naive state. 


\section{A.1. MODEL A}

In model $\mathrm{A}$, we describe the $\mathrm{CD} 8$ response by a partial differential equation. We define $y(t, \tau)$ as the number of responding cells per day at time $t$ that were recruited $\tau$ days earlier (we refer to $\tau$ as the age of the cells). The immune "program" is described by a function $F(\tau)$. In order to get an expansion phase followed by a contraction phase we use a function $F(\tau)$ that is initially positive (and equal to the rate of expansion), and is subsequently negative (and equal to the rate of contraction). Subsequent to both expansion and contraction phases the population is assumed to remains stable.

$$
\text { Naive : } \frac{\mathrm{d} N(t)}{\mathrm{d} t}=-b N(t) P(t),
$$

Total response : $\quad Y(t)=\int_{\tau=0}^{t} y(t, \tau) \mathrm{d} \tau$,

$$
\begin{gathered}
\text { Recruitment : } \quad y(t, 0)=-\frac{\mathrm{d} N(t)}{\mathrm{d} t}, \\
\frac{\partial y(t, \tau)}{\partial t}+\frac{\partial y(t, \tau)}{\partial \tau}=F(\tau) y(t, \tau) .
\end{gathered}
$$

The strict program is described by the function $F(\tau)$, which gives post-stimulation dynamics of a CD8 cell and its progreny. We note that the dynamics of the CD8 response in fact largely specifies the necessary form of this function $F(\tau)$. Since these dynamics begin with rapid proliferation, proceed to a death phase and conclude with a memory phase (Fig. 1), we expect $F(\tau)$ to begin at the maximal rate of proliferation (observed during the expansion phase), to become negative during the contraction phase, and to be close to zero during the memory phase. In Fig. A1 we plot a representative function used for $F(\tau)$. We have also explored other functions for $F(\tau)$, including a stepwise function which jumps from a fixed positive value during the expansion phase to a fixed negative value during the contraction phase and finally to zero during the memory phase in a manner similar to that employed by De Boer et al. (2001). We choose a smooth function plotted in Fig. A1 (which is composed of added Hill functions mentioned in Section A)

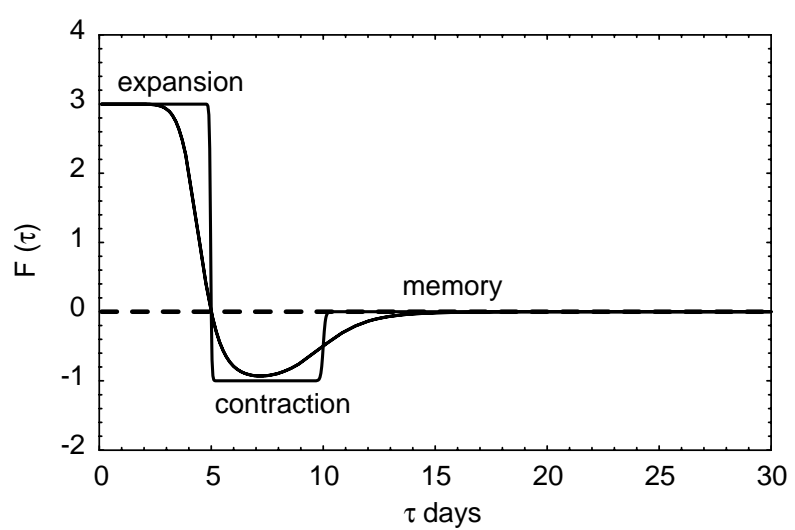

FIG. A1. Antigen-independent proliferation program. The function $F(\tau)$ which describes the antigen-independent dynamics of the cell population in terms of the rate of proliferation of immune cells as a function of time $\tau$. As described in the paper this function should first be positive, then negative and finally close to zero in the expansion contraction and memory phases of the response. The data on the dynamics of the response are not sufficiently detailed to allow us to determine the details of this function such as whether it is a smooth function or a step function indicated above.

simply because we expect the transition in growth rates defined by the program to be continuous (see Section A). We note that the data available (Fig. 1) are not sufficiently detailed to allow us to discriminate between these alternative functional forms for $F(\tau)$.

Thus properties of a cell change with the age of the cell $\tau$. We would expect that initially the cells rapidly gain effector function and that this is subsequently lost as the cells gain memory functions. The model thus naturally describes the transition between effector and memory cells. For simplicity in the first models the cells are assumed to be effector cells prior to time $\tau^{*}$ and subsequently memory cells. Thus

$$
\begin{gathered}
\text { Effector : } \quad E(t)=\int_{\tau=0}^{\tau^{*}} y(t, \tau) \mathrm{d} \tau, \\
\text { Memory : } \quad M(t)=\int_{\tau=\tau^{*}}^{\infty} y(t, \tau) \mathrm{d} \tau,
\end{gathered}
$$

As is conventional in models of $\mathrm{T}$ cell responses (Nowak \& Bangham, 1996; DeBoer \& Perelson, 1995; Pilyugin et al., 1997; Wodarz et al., 1998, 2000; DeBoer et al., 2001), we assume that the pathogen density (or the number 
of infected cells) increases at some initial rate $r$ (gradually slowing as a carrying capacity $c$-imposed by, for example, target cell limitation - is reached). Pathogens (or infected cells) are killed at a rate proportional to the product of pathogen density and effector cell density. Thus the equation governing pathogen dynamics is:

$$
\begin{aligned}
& \text { Pathogen : } \frac{\mathrm{d} P(t)}{\mathrm{d} t} \\
& =r P(t)\left(1-\frac{P(t)}{c}\right)-h P(t) E(t) .
\end{aligned}
$$

\section{A.2. MODEL B}

In model $\mathrm{B}$, we describe the $\mathrm{CD} 8$ response by a system of ordinary differential equations. Let $y_{n}(t)$ equal the number of cells which have undergone $n$ divisions. Naive cells $N$ are taken as cells having undergone 0 divisions. The basic structure of this model is similar to the models for the ageing of immune responses (Antia et al., 1996; Pilyugin et al., 1997), except that it includes antigen-independent proliferation.

$$
\text { Naive : } \frac{\mathrm{d} N(t)}{\mathrm{d} t}=-b N(t) P(t),
$$

Recruited : $\frac{\mathrm{d} y_{1}(t)}{\mathrm{d} t}=b N(t)-k_{1} y_{1}(t)-d_{1} y_{1}(t)$,

$$
\text { (division } n) \quad \begin{aligned}
\frac{\mathrm{d} y_{n}(t)}{\mathrm{d} t} & =2 k_{n} y_{n-1}(t)-k_{n} y_{n}(t) \\
& -d_{n} y_{n}(t), \quad n=2,3,4, \ldots .
\end{aligned}
$$

During the first $a$ divisions, proliferation occurs: the birth rate exceeds the death rate $\left(k_{n}>d_{n}\right)$. For the subsequent $b$, divisions contraction takes place: the death rate exceeds the birth rate $\left(k_{n}<d_{n}\right)$. Subsequently, during the memory phase, the birth rate equals the death rate $\left(k_{n}=d_{n}\right)$.

In this model, the properties of a cell change with the number of divisions the cell has undergone $n$. We would expect that initially the cells rapidly acquire effector function with division and that this is subsequently lost as the cells acquire memory functions. For simplicity, we assume cells to be effector cells when the number of divisions $n<(a+b)$, and they are subsequently memory cells. Thus

$$
\begin{aligned}
& \text { Effector: } \quad E(t)=\sum_{n=1}^{a+b-1} y_{n}(t), \\
& \text { Memory : } \quad M(t)=\sum_{n=a+b}^{\infty} y_{n}(t) .
\end{aligned}
$$

As in Model A, the dynamics of the pathogen are given by

Pathogen : $\frac{\mathrm{d} P(t)}{\mathrm{d} t}=r P(t)\left(1-\frac{P(t)}{c}\right)-h P(t) E(t)$.

\section{A.2.1. Extension to Multiple Epitopes}

In this section we describe the multi-epitope version of Model A. A similar approach can be used for Model B. As in the single-epitope version, $N_{i}(t)$ and $Y_{i}(t, \tau)$ are the populations of naive and recruited cells of age $\tau$ specific for epitope $i$ at time $t$.

$$
\text { Naive : } \frac{\mathrm{d} N_{i}(t)}{\mathrm{d} t}=-b_{i} N_{i}(t) P(t)
$$

$$
\text { Response : } \quad Y_{i}(t)=\int_{0}^{\infty} y_{i}(t, \tau) \mathrm{d} \tau,
$$

$$
\frac{\partial y_{i}(t, \tau)}{\partial t}+\frac{\partial y_{i}(t, \tau)}{\partial \tau}=F(\tau) y_{i}(t, \tau)
$$

$$
y_{i}(t, 0)=-\frac{\mathrm{d} N_{i}(t)}{\mathrm{d} t}
$$

Effector : $\quad E_{i}(t)=\int_{\tau=0}^{\tau^{*}} y(t, \tau) \mathrm{d} \tau$,

$$
\text { Memory : } \quad M_{i}(t)=\int_{\tau=\tau^{*}}^{\infty} y(t, \tau) \mathrm{d} \tau \text {. }
$$

In a simple extension of the previous models, the pathogen dynamics are given by the 
following equation:

$$
\text { Pathogen : } \begin{aligned}
\frac{\mathrm{d} P(t)}{\mathrm{d} t} & =r P(t)\left(1-\frac{P(t)}{c}\right) \\
& -\sum_{i} h_{i} E_{i}(t) P(t) .
\end{aligned}
$$

\section{A.2.2. Parameters for Figures}

We chose parameters in agreement with the estimates obtained by DeBoer et al. (2001). For Fig. 3 we use Model A. (Similar results are obtained using Model $\mathrm{B}$, with parameters $r=5$, $c=10^{6}, h_{1}=10^{-4}, h_{2}=3 \times 10^{-5}, b_{1}=5 \times 10^{-4}$ and $\quad b_{2}=10^{-4}, \quad N_{1}(0)=30, \quad N_{2}(0)=10$, $P(0)=10)$. The function $F(\tau)$ describing the antigen-independent program is shown in Fig. A1. As described in the text we have used a number of forms for this function including a piecewise continuous (step) function and a continuous function. The particular continuous function chosen for the simulations (used because as $n \rightarrow \infty$ it approximates to a step function) has the form

$$
\begin{aligned}
F(\tau) & =s_{e}-\left(s_{e}+s_{c}\right)\left(\frac{\tau^{n}}{\left(s_{c} / s_{e}\right) T_{e}^{n}+\tau^{n}}\right) \\
& +s_{c}\left(\frac{\tau^{n}}{\left(T_{e}+T_{c}\right)^{n}+\tau^{n}}\right) .
\end{aligned}
$$

Parameters $n=10, s_{e}=3.0, s_{c}=0.5, T_{e}=6.5$, $T_{c}=8$.

In Fig. 4 during the antigen-dependent expansion phase, the rate of proliferation equals $P /(k+P)$, with $k_{1}=10^{3}$ and $k_{2}=3 \times 10^{3}$ for the two epitopes. For the external signal model [Fig. 4(A)] the window of antigen-dependent proliferation has duration 2.5 days following infection. For the internal signal model [Fig. 4(B)] the window of antigen-dependent proliferation is for a duration of 1.1 days following stimulation of the cell. 